\title{
Configurable Text Stamp Identification Tool with Application of Fuzzy Logic
}

\author{
J. He and Andy C. Downton \\ Department of Electronic Systems Engineering, University of Essex, UK \\ $\{j h e, a c d\} @ e s s e x . a c . u k$
}

\begin{abstract}
This paper presents our work on locating and removing unwanted text stamps within archive documents which are being prepared for OCR. Text stamps mainly comprise one or several text lines with a fixed shape, font size and colour, and may appear anywhere on the document with variable orientation and overlap of other text fields. We apply a configurable user interface to register features of a sample stamp (such as corners, font-size and print colour) as a template using fuzzy rules, and then analyse each document image to find matching stamps using fuzzy functions as a classification mechanism. The configurable interface allows the user to decide which and how many features should be used to describe the target stamp. Evaluation was very encouraging. We tested 1,241 specimen index cards from a biological archive card index, and achieved $92-95 \%$ correct detection rate and $85-95 \%$ complete removal rate.
\end{abstract}

\section{Introduction}

Identification of fixed symbols (e.g. seal imprints, logos and signatures) attracts a lot of attention in the field of document image analysis. [1] [2] [3] [4] present several methods of identifying seal imprints, while [5] [6] [7] [8] present approaches for verifying signatures. [9] and [10] provide different ways of recognizing logos. Most methods follow three steps: template registration, detection and classification.

- Template registration records modeled features of the target symbol. Feature modeling varies widely. [1] [2] [3] [4] [5] [6] [7] [9] [10] calculate a set of constraints for features by using formulae. [11] uses a neural network to train a set of weights for features. [8] uses fuzzy sets to model features.

- Detection aims to distinguish the potential target symbol from other content of the document. In most document images, symbols are mixed with other components e.g. text. Therefore, some measures have to be taken to narrow the target. [4] suggests a method using color information to separate signatures and seal imprints from bank check backgrounds. [10] suggests using the size of the symbol block to reduce the potential candidates.

- Finally, classification is the matching process, which compares the candidate with the template.

We are currently analysing archive documents, such as specimen index cards and library index cards. These frequently contain stamps, consisting of several text lines with 
specific shape, font size and color. These stamps are not useful elements of the archive documents, and should be removed to reduce errors in extracting useful textual information. In this paper, we suggest a method to identify stamps by using fuzzy logic [12] [13]. We use fuzzy terms to model features of corners of the stamp with a configurable user interface, and then use the method suggested in [10] to narrow down the target document areas. Finally, we match the candidate with the template by using a fuzzy inference mechanism to overcome variations in printing of the stamp.

Our system has several advantages. It is flexible and adaptable for different applications and datasets, as the configuration and features of each stamp to be detected are defined visually by the user, and then mapped to fuzzy rules, rather than hardprogrammed. By using fuzzy logic, parameterised via the user interface, the system can handle stamps and document sets with widely differing formats and characteristics reliably.

The paper is organised as follows: Section 2 starts by reviewing our overall system, and then outlines the features we extract to help identify and locate the stamps. Section 3 describes our segmentation method. Section 4 describes how configurable templates are constructed by users of the system, and how template attributes are mapped to fuzzy logic parameters. Section 5 explains how the fuzzy logic parameters are matched against features extracted from test archive images, enabling the presence of a stamp to be detected and the stamp removed. Section 6 describes how the system was evaluated, and presents the results obtained. Section 7 draws conclusions and discusses further work.

\section{System Overview and Stamps Feature Analysis}

\subsection{System Overview}

Index cards have a consistent physical format (typically $5^{\prime \prime} \times 3^{\prime \prime}$ or $6^{\prime \prime} \times 4^{\prime \prime}$ thin card) but a wide range of handwritten, typed, printed and mixed text content. Fig. 1 shows a variety of formats found within the index cards at the UK Natural History Museum (NHM), which range from highly-structured to relatively free-format text. More than 500,000 cards have now been digitized using a scanner system first discussed in [14], and are progressively being made accessible online [15]. OCR of the content of these cards varies from difficult (where high quality typed or printed text is used, but with specialized scientific lexicons and non-standard abbreviations) to extremely challenging (similar lexicons but handwritten and amended text). Although the overall variation over a complete dataset (hundreds of thousands of cards) is large, substantial batches of cards within the dataset are often similarly constructed, making it possible for a taxonomist to improve OCR by defining batch-specific card formats using a few user-defined templates. Processing then involves anything from recognizing a batch containing a single format of card (and single corresponding card template), through to processing a mixed batch of cards with several corresponding templates. Our system is therefore designed to support the process of user-assisted template configuration for card index document analysis and recognition. An important sub-component of this system identifies and removes validation stamps, which are found on many index cards but do not contribute to the useful textual content of the card. The stamp identification and removal system comprises four parts, segmentation, template registration, identification and removal. 


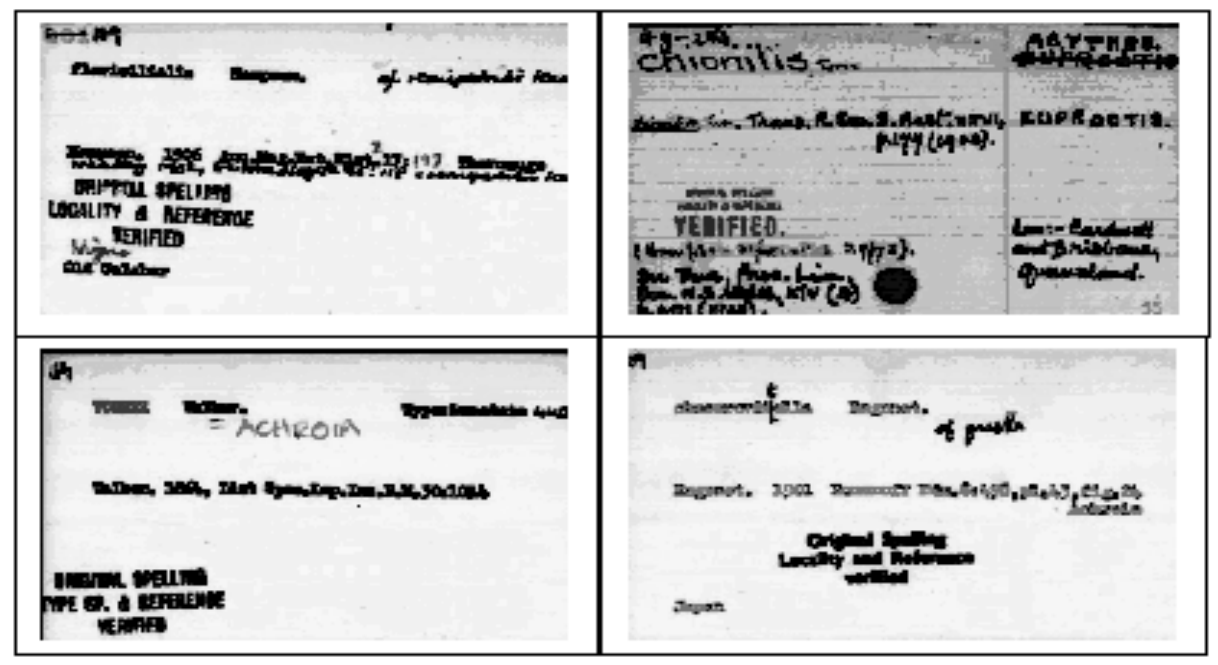

Fig. 1. Example card formats from the Natural History Museum archive card index, showing four commonly encountered validation stamps.

\subsection{Feature Extraction}

When humans try to identify a document pattern, the global features first attract their attention and then the local features. For archive validation stamps, the global features are generally colour and structure, and the local features are the textual content. In this research, we identify stamp patterns on archive cards by feature extraction of global colour and structure.

\subsection{Stamp Colour Analysis}

Validation stamps on archive cards are produced by pressing the stamp onto an inkpad and then onto the card. Therefore stamps images are normally a single colour (black, blue, red or green) according to the colour of the inkpad. If the inkpad colour differs from the predominant text colour of the archive card, then simple colour-based segmentation of the elements of the card may be sufficient to identify the stamp uniquely, enabling it to be removed from the remainder of the card content. Commonly however, both the stamp and the typed or handwritten card text use the same colour ink, making structural analysis necessary to identify the stamp pattern, even when colour segmentation has been applied to simplify structural analysis. We discuss colour map detection for archive documents in a separate paper submitted to DAS2004 [16].

\subsection{Stamp Structure Analysis}

Four different validation stamp styles have been found in our archive documents, as shown in Fig. 2.

We concluded that the global structure of these stamp patterns has two elements, inner structure and outermost boundary. The inner structure depicts the inner skeleton 


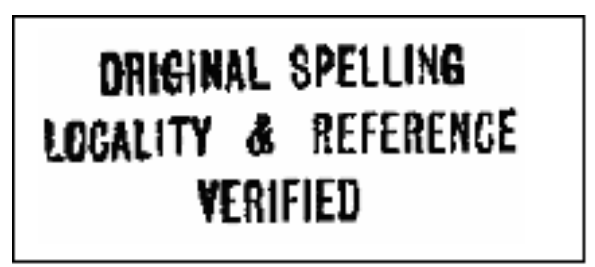

(a)

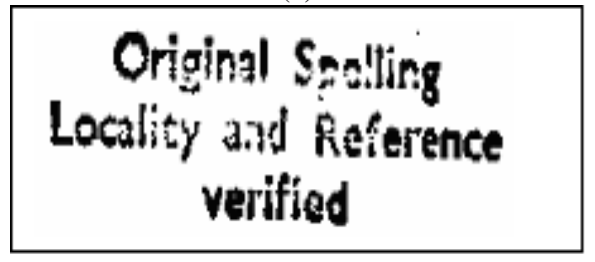

(c)

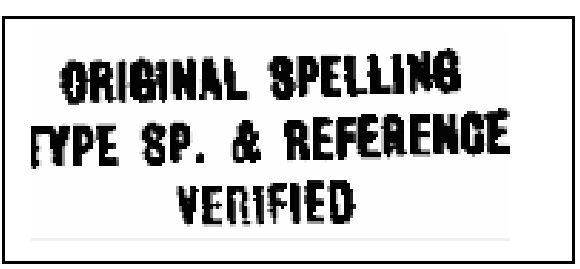

(b)

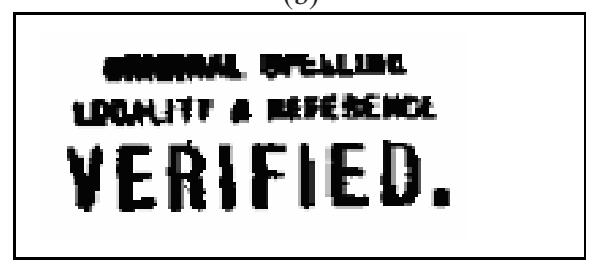

(d)

Fig. 2. NHM validation stamps (stamps (a) and (d) are common, stamps (b) and (c) rare, so we evaluated our system only using (a) and (d)).

of the patterns, which is represented by horizontal white spaces between text lines and vertical white spaces between words. The outermost boundary reflects patterns' shape and size, where the shape depicts the outermost edges while the size indicates their dimensions. However, by investigating many archive documents, we found that the inner structure is not reliable, as it often suffers from "inner damage" (due to the mechanism of pressing the stamp onto the card by hand) as shown in Fig. 3.

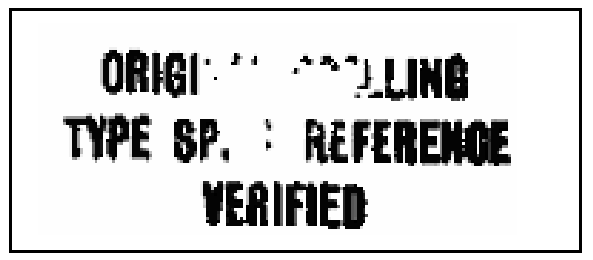

(a)

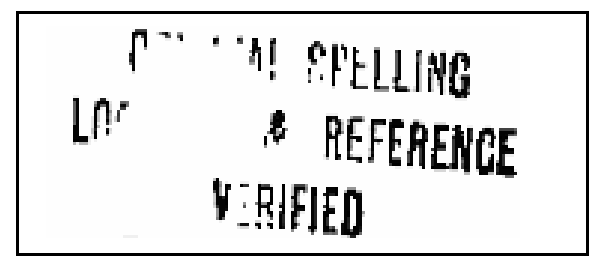

(b)

Fig. 3. Examples of inner structure damage to NHM validation stamps.

Therefore we concentrated our efforts on utilising features from the outermost boundary, because it is more reliable. The stamp patterns hence can be represented by a polygon contour where corner points correspond to the ends of text lines. The lengths (e.g. D1_2) and relative orientations (represented by internal corner angles, e.g. A3_1_2) of the sides of the enclosing polygon are sufficient to define the polygon and thus the outer boundary shape and size of the stamp. Fig. 4 shows the boundary contour and identified features for the stamp pattern shown in Fig. 2(a). A single feature, skew angle, is sufficient to define the absolute rotational orientation of the stamp, which because of its mechanism of formation, is often not printed squarely on the archive card (in rare cases, it is even printed upside down). Finally, the variation in the fonts, fontsizes 
and capitalization of the characters within each validation stamp can be represented by a "font height" feature (e.g. F1) recorded for each corner of the stamp. Here, we use the character height of the character at each corner rather than its width, because a font width feature would require accurate horizontal segmentation of characters, whereas font height only requires segmentation from non-overlapping adjacent lines.

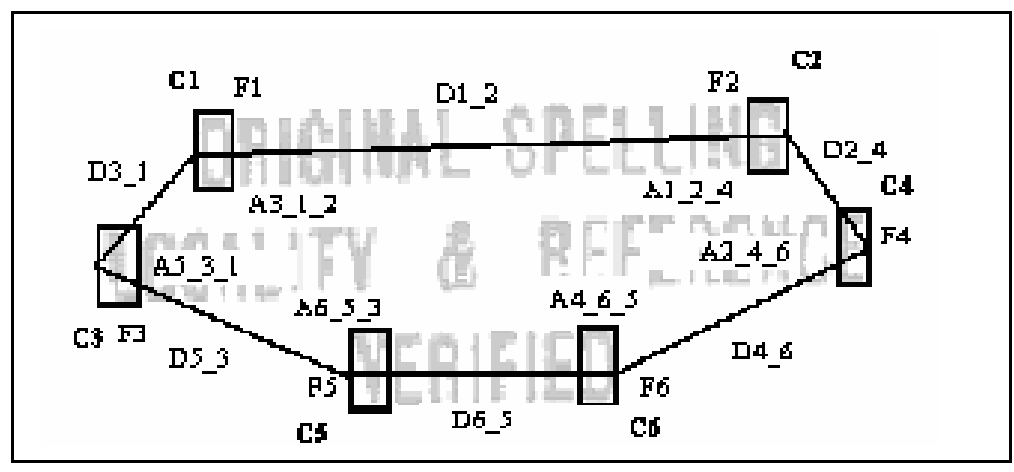

Fig. 4. Overview of configurable validation stamp removal system.

\section{Stamp Structure Analysis}

Each text string on an archive card is considered as a fundamental element, which contains structural features. The purpose of stamp structure analysis is to segment all the text strings from the stamp color plane obtained previously, and then extract corner features from each text string. First of all binarization is applied to the stamp colour plane(Fig. 5(a)). Then binary connected component bounding boxes are used to group the foreground pixels of the image into features. No filtering or smearing is applied before binary connected component analysis, hence the bounding boxes obtained will correspond to individual characters or groups of touching characters, as shown in Fig. 5(b). To avoid black pepper noise, we then apply a filter to remove bounding boxes smaller than 3 pixels square. The filter is adjustable according to the application through the interface. Adjacent bounding boxes are then accumulated to generate short text strings, representing words or phrases. Two situations can arise in forming the text strings. The simplest case is when the stamp colour plane contains only strings corresponding to the stamp image, because all other text strings are contained in a different colour plane. Commonly however, text strings may or may not belong to the stamp, because the stamp printing is the same colour as other text in the archive image. In this latter case, all the short text strings, and all their corresponding potential corners, need to be identified, and the positional relationships between text corners are then used to identify and eliminate string configurations which can not represent stamps.

A short text string is accumulated from left to right. If the horizontal distance between two bounding boxes is less than twice the initial character width of that string and they overlap vertically by more than half the initial character height, we consider that these two bounding boxes are adjacent to each other. The initial values for character 


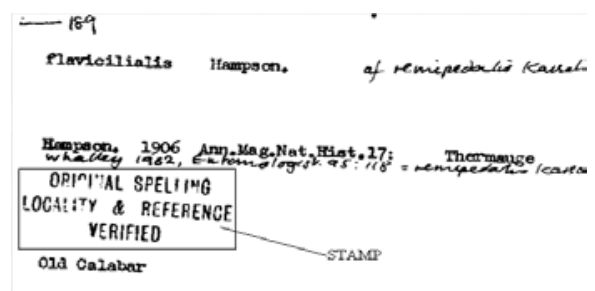

(a)

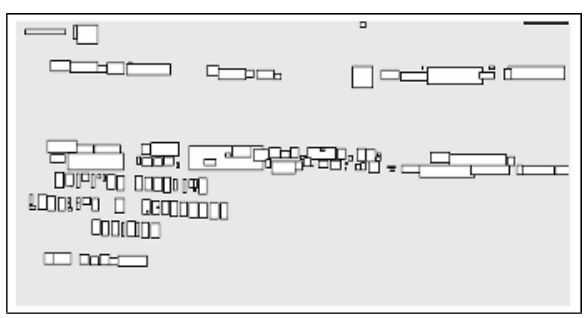

(b)

Fig. 5. Examples of binarized stamp colour plane (a) and its connected components (b).

width and height are successively replaced by an average of those of previously joined bounding boxes, allowing the assumed character size to be progressively updated along a text string. The process will be terminated when no further adjacent bounding box is found. Then a new short string is initialized from the nearest bounding box. Repeating the whole process left-to-right and top-to-bottom, finally we have a set of short strings. The short strings are then further joined into longer strings by using a Hough Transform [17] [18]. The ends of the long strings are the identified "corners" of text lines, which are the primary feature used to identify instances of stamp templates occuring in each archive card image.

\section{Stamp Template Registration}

In this section, we indicate how the template for a stamp is constructed by using fuzzy logic to represent its structural features. First of all, the user selects a sample card image containing a representative sample of the stamp pattern using the system's graphical user interface [19]. This stamp image is then cropped and converted into a binary image. Then, using the segmentation method described in Section 3 to obtain each text line of the stamp, the "corners" of the stamp are located. From the structure analysis in Section 2.4, three structural features, angle, distance and font, are identified for each "corner".

\subsection{Structural Features Registration}

For feature angle, each inward angle of a stamp can be measured by drawing a closed polygon graph connecting all the corners of the template stamp. The average angle, Aaver $=180 \times(\mathrm{n}-2)$, depends on $\mathrm{n}$, the number of sides the stamp contour contains. The maximum Amax, minimum Amin and average corner angle Aaver are then used to scale the allowed fuzzy corner angle ranges of the stamp. Based on these values, a membership function for feature angle is defined using three ranges, "SMALL", "MEDIUM" and "LARGE". The peaks of each range correspond to Amin, Aaver and Amax respectively, and any angle whose value is outside the range [Amin, Amax], is considered as "NULL". In the case of stamp Fig. 2(a), the membership function is defined as shown in Fig. 6(a), where Amax=180, Amin=60 and Amax= 120. Similarly, the membership function for features distance and font are also defined with three ranges, "SHORT", 


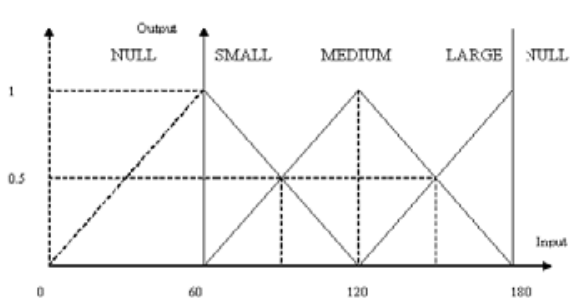

(a)

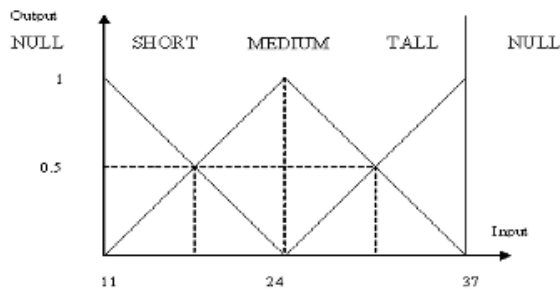

(c)

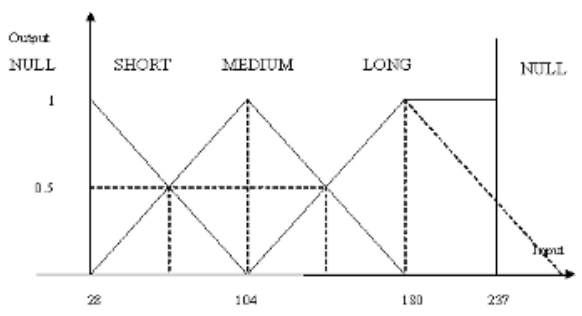

(b)

Fig. 6. Examples of membership function for feature angle (a) distance (b) and font(c).

Table 1. Example of template expression for stamp structural features angle, distance and font.

\begin{tabular}{|c|c|c|c|c|c|c|}
\hline Features & C1 & C2 & C3 & C4 & C5 & C6 \\
\hline Angle & A3_1_2 & A1_2_3 & A5_3_1 & A2_4_6 & A6_5_3 & A4_6_5 \\
\hline Fuzzy expression & MEDIUM & MEDIUM & SMALL & SMALL & LARGE & LARGE \\
\hline Distance & D1_2 & D2_4 & D4_6 & D6_5 & D5_3 & D3_1 \\
\hline Fuzzy expression & LONG & SHORT & MEDIUM & MEDIUM & MEDIUM & SHORT \\
\hline Font & F1 & F2 & F3 & F4 & F5 & F6 \\
\hline Fuzzy expression & MEDIUM & MEDIUM & MEDIUM & MEDIUM & MEDIUM & MEDIUM \\
\hline
\end{tabular}

"MEDIUM", "LONG" and "SHORT", "MEDIUM", "TALL". In the case of stamp Fig. 2(a), the membership functions for these features are shown as Figures 6(b) and (c) respectively.

Using the membership function, the measured values of angle, distance and font for any configuration of corners corresponding to a possible stamp can be mapped into their corresponding fuzzy representation. The template fuzzy expression for stamp Fig. 2(a) is, for example, represented as Table 1.

\subsection{Registration of Other Important Stamp Parameters}

Beside the structural features, there are some other important global stamp parameters; these are the overall size of a stamp $\operatorname{SZt}(x, y)$ (in pixels) and maximum vertical WHPy and horizontal WHPx inner white space (in pixels). These parameters are used for stamp isolation, as mentioned in the next section. 


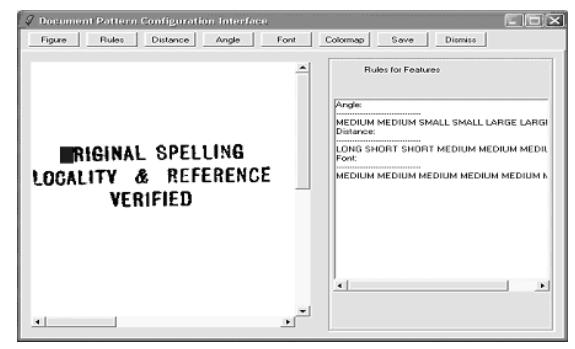

Fig. 7. Stamp template configuration interface.

\section{DRIGINAL SPELLIKG SHIIT' \& REFERENOE VERIFIED}

Fig. 8. Example of stamp.

\subsection{Template Configuration}

All the corner features (angle, distance and font) and global parameters are visually identified and represented on the graphical user interface Fig. 7. Curators can therefore easily understand both the graphical significance and linguistic representation of each feature. This allows curators to change the attributes of each feature and parameters so that the stamp removal system can readily be reconfigured for use with other stamps having different formats and atributes.

\section{Identification and Removal}

\subsection{Stamp Isolation}

Through colour segmentation, the stamp colour plane can be obtained. The colour plane is then binarized. In order to improve analysis accuracy and reduce complexity, we use the method suggested by [10], in which a binary connected component bounding box with smear values (WHPy and WHPx) is used to locate potential stamp blocks. In our application, if the size of the located block $\operatorname{SZc}(x, y)$ is similar to or bigger than that of the registered $\operatorname{SZt}(x, y)$, it is considered as a candidate stamp block. We then carry out stamp structure analysis on these isolated candidate stamp blocks to extract all the "corners" they contain, as described previously in Section 3.

\subsection{Matching}

The extracted "corners" are grouped in the same format as has been registered for each template. Features of each group can be extracted, and then converted into their corrsponding fuzzy representation by using the previously defined membership functions (e.g. fig. 6(a)(b)(c)). For instance, a group of "corners", which is extracted from Fig. 8, has a set of structural features as shown in Table 2. We construct a fuzzy inference mechanism to map these instance features to those registered for each template by deriving a set of fuzzy "IF-THEN" rules.

The inference mechanism provides a weighting system to weight each of the features. For instance, for the feature angle, if an angle is classified as "SMALL" and its counterpart in the template is "LARGE", it weighs $0 \%$; if classified as "MEDIUM", it 
Table 2. Instance of fuzzy expression for a group of corners with structural features angle, distance and font.

\begin{tabular}{|c|c|c|c|c|c|c|}
\hline Features & C1 & C2 & C3 & C4 & C5 & C6 \\
\hline Angle & LARGE & MEDIUM & NULL & SMALL & LARGE & LARGE \\
\hline Distance & LONG & SHORT & NULL & MEDIUM & NULL & SHORT \\
\hline Font & MEDIUM & MEDIUM & MEDIUM & MEDIUM & MEDIUM & MEDIUM \\
\hline
\end{tabular}

Table 3. Weighting table for feature angle.

\begin{tabular}{|c|c|c|c|}
\hline Angle & SMALL & MEDIUM & LARGE \\
\hline SMALL & $100 \%$ & $50 \%$ & $0 \%$ \\
\hline MEDIUM & $50 \%$ & $100 \%$ & $50 \%$ \\
\hline LARGE & $0 \%$ & $50 \%$ & $100 \%$ \\
\hline NULL & $0 \%$ & $0 \%$ & $0 \%$ \\
\hline
\end{tabular}

weighs 50\%; if classified as "LARGE", it weighs $100 \%$; if classified as "NULL", it also weighs $0 \%$. Table 3 shows the full weighting table for feature angle. Other features can also be weighted in the same way, therefore the example of Table 2 will be weighted as shown in Table 4. The weight for each "corner" $C i(A, D, F)$ is the average of the weights of its angle $A(i)$, distance $D(i)$ and font $F(i)$. The overall weight $S(C 1, \ldots, C i)$ for the group of "corners" is the average of weight of $C 1(A, D, F), \ldots, C i(A, D, F)$.

Table 4. Example of weighting for a group of corners structural features angle, distance and font.

\begin{tabular}{|c|c|c|c|c|c|c|}
\hline & C1 & C2 & C3 & C4 & C5 & C6 \\
\hline Angle & $50 \%$ & $100 \%$ & $0 \%$ & $100 \%$ & $100 \%$ & $100 \%$ \\
\hline Distance & $100 \%$ & $100 \%$ & $0 \%$ & $100 \%$ & $0 \%$ & $100 \%$ \\
\hline Font & $100 \%$ & $100 \%$ & $100 \%$ & $100 \%$ & $100 \%$ & $100 \%$ \\
\hline Weight & $83.3 \%$ & $100 \%$ & $33.3 \%$ & $100 \%$ & $66.7 \%$ & $100 \%$ \\
\hline Overall weight & \multicolumn{5}{|c|}{$483.3 \% / 6=80.55 \%$} \\
\hline
\end{tabular}

If any group of corners is classified as "HIGH" (>75\%) and its overall weight is the highest of all candidate stamps, this group of "corners" is considered to be the "corners" of the stamp. The stamp is therefore detected.

\subsection{Stamp Removal}

The output of the inference mechanism locates a group of "corners" which represents the detected stamp. Using the known position of the stamp's corners, the maximum boundary of each text line of the stamp is easily located (each text line contains two corners, one at each end). The stamp can thus be removed by deleting all the pixels between each pair of corners from the input image, and (optionally) replacing them with background pixels (depending on what subsequent processing is required). 


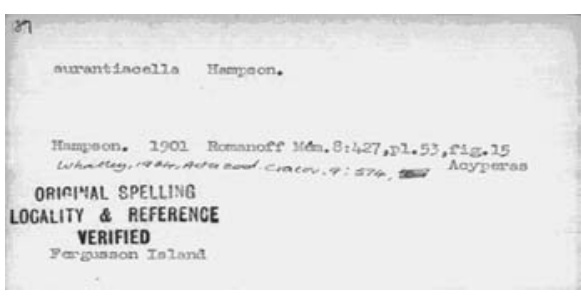

(a)

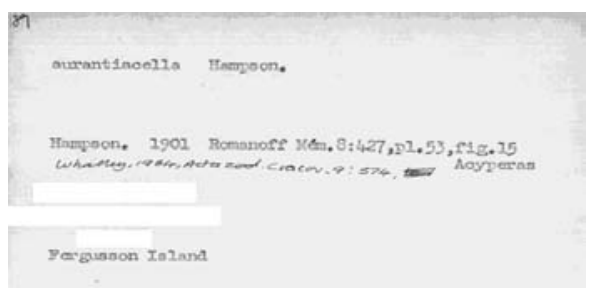

(b)

Fig. 9. Example of input image with Type I stamp (a), stamp removed (b).

\section{Evaluation and Results}

\subsection{Evaluation}

Through investigation, we found that over $90 \%$ of archive cards from the Natural History Museum contain mainly two types of stamp patterns (Figures 3(a) and (d)). Therefore, we concentrated our evaluation on these two stamp types. Two sets of specimen index cards from the Natural History Museum were chosen for evaluation. The first set has 545 cards, 269 of which contain type I stamps (Fig. 2(a)), where the stamp is the same colour as most of other text (e.g. text which is black) on the card image. The second set has 676 cards, 513 of which contain type II stamps (Fig. 2(d)), which are of a different colour (e.g. red) compared with the remaining card image text.

Table 5. Result of evaluation.

\begin{tabular}{|c|c|c|c|c|c|}
\hline Stamp Type & No. of images & No. of stamps & Correctly detected & Wrongly detected & Complete removed \\
\hline Type I & 545 & 292 & $269 / 292(92.1 \%)$ & $1 / 545(0.2 \%)$ & $250 / 269(85.6 \%)$ \\
\hline Type II & 676 & 513 & $487 / 513(94.9 \%)$ & $1 / 676(0.1 \%)$ & $487 / 513(94.9 \%)$ \\
\hline
\end{tabular}

\subsection{Results}

The results shown in Table 2 are encouraging. 92.1\% of Type I stamps were successfully detected and $85.6 \%$ of them were completely removed (Fig. 9(b)), while $94.9 \%$ of Type II stamps were successfully detected and all those detected were removed completely. The undetected errors for Type I stamps were caused either by serious fading (where most of the stamp has been lost, Fig. 10(a)) or where the stamp touches other text, Fig. 10(b). The undetected errors for Type II stamps were only caused by serious colour fading; as a result, the stamp colour plane couldn't be separated properly in the first place, hence resulting in a damaged stamp. Some useful text is inevitably removed with the stamps in cases where the stamp touches other text of the same colour as shown in Fig. 11. These situations are classified as incomplete removal, and result in the figures for stamp removal being lower than those for stamp detection for Type I stamps. Not surprisingly, the overall detection and removal rate for Type II stamps is higher than Type I, because Type II stamps are usually separated from any adjacent text as part of the initial colour plane segmentation. 


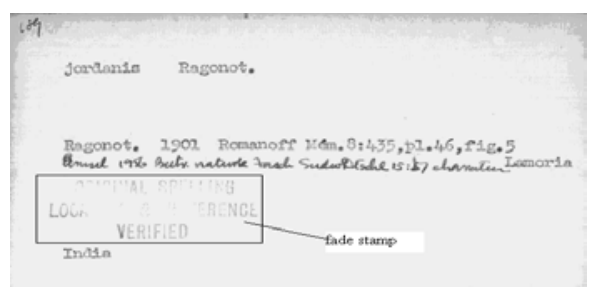

(a)

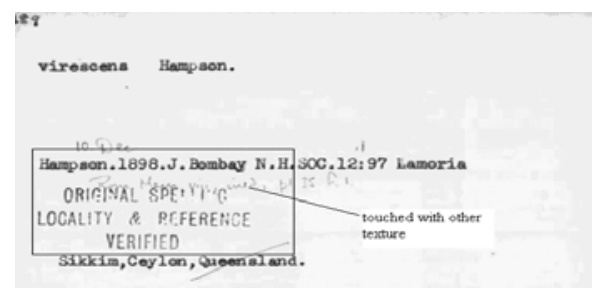

(b)

Fig. 10. Example of (a) faded Type I stamp, (b) Type I stamp touching other text.

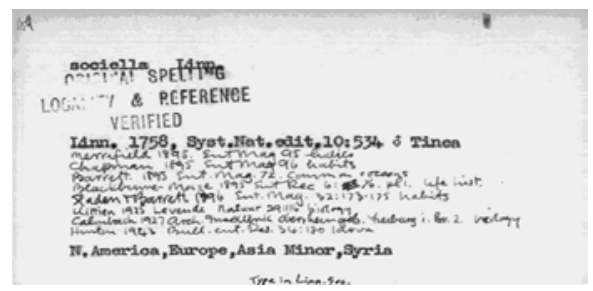

(a)

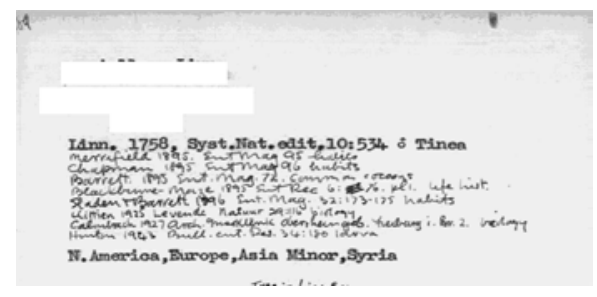

(b)

Fig. 11. Example of Type I stamp (a) touching other text, (b) text has been removed with the stamp.

\section{Conclusion}

In this paper, the use of fuzzy logic to model structural features of text stamps has been proposed. With fuzzy modeled features, the stamp can be detected in spite of many variations or defects in the features extracted (e.g. partial damage, shape transformation). The application of a configurable interface for template enhance the flexibility

of the system and makes it adaptable for processing widely varying batches of archive images. The evaluation results are very encouraging, with $92 \%-95 \%$ correct stamp detection rate and $85 \%-95 \%$ complete removal rate being achieved.

\section{References}

1. S.W.Lee, J.H.Kim: Unconstrained seal imprint verification using attributed stroke graph matching. Pattern Recognition 22 (1989) 653-664

2. K.Ueda: Automatic seal imprint verification system with imprint quality assessment function and its performance evaluation. IEICE Trans.Inf. and Syst. E-77D (1994) 885-894

3. R.Haruki, T.Horiuchi, H.Yamada, K.Yamamoto: Automatic seal verification using threedimensional reference seals. Proc. ICPR'96 13th Int.Conf. on Pattern Recognition Vol.1 (1996) 199-203

4. K.Ueda, T.Mutoh, K.Matsuo: Automatic verification system for seal imprints on japanese bankchecks. Proc. ICPR'98 14th Int.Conf. on Pattern Recognition Vol.1 (1998) 629-632

5. R.Plamondon, G.Lorette: Automatic signature verification and writer recognition -the state of the art-. Pattern Recognition 22 (1989) 107-131

6. B.Wirtz: Stroke-based time warping for signature verification. Proc.ICDAR'95 the Third Int.Conf.on Document Analysis and Recognition Vol.1 (1995) 179-182 
7. C.Schmidt, K.F.Kraiss: Establishment of personalized templates for automatic signature verification. Proc.ICDAR'97 4th Int.Conf.on Document Analysis and Recognition Vol.1 (1997) 263-267

8. M.Hanmandlu, K.R.Murali.Mohan, S.Chakraborty, G.Garg: Fuzzy modeling based signature verification system. Proc.ICDAR'01 6th Int.Conf.on Document Analysis and Recognition (2001) 110-114

9. D.s.Doerman, E.Rivlin, I.Weiss: Logo recognition using geometric invariants. Proc. ICDAR'93 2nd Int.Conf.on Document Analysis and Recognition (1993) 894-897

10. P.Suda, C.Bridoux, B.Kammerer, G.Maderlechner: Logo and word matching using a general approach to signal registration. Proc.ICDAR'97 4th Int.Conf.on Document Analysis and Recognition Vol.1 (1997) 61-65

11. T.Cheng, J.Khan, H.Liu, D.Y.Y.Yun: A symbol recogonition system. Proc.ICDAR'93 2nd Int.Conf.on Document Analysis and Recognition (1993) 918-921

12. L.A.Zadeh, J.Kacprayk: Fuzzy Logic for the Management of Uncertainty. John-Wiley, New York (1992)

13. D.Dubois, H.Prade, R.Y.Yager: Fuzzy Sets for Intelligent Systems. Morgan Kaufmann Publishers, San Mateo (1993)

14. A.C.Downton, A.C.Tams, G.J.Wells, A.C.Holmes, S.M.Lucas: Constructing web-based legacy index card archives- architectural design issues and initial data acquisition. Proc.ICDAR'01 6th Int.Conf.on Document Analysis and Recognition (2001) 854-858

15. (http://www.nhm.ac.uk/entomology/lepindex/)

16. J.He, A.C.Downton: Colour map detection for archive documents. submitted to DAS2004 (2004)

17. S.C.Hinds, J.L.Fisher, D.P.D'Amato: A document skew detection method using run-length encoding and the hough transform. Proc. 10th Int'1 Conf. Pattern Recognition(ICPR), IEEE CS Press, Los Alamitos, Calif., (1990) 464-468

18. J.Illingworth, J.Kittler: A survey of the hough transform. Computer Graphics and Image Processing Vol.44 (1988) 87-116

19. J.He, A.C.Downton: User-assisted archive document image analysis for digital library construction. Proc.ICDAR'03 7th Int.Conf.on Document Analysis and Recognition Vol.1 (2003) 498-502 